\title{
Disambiguating Preferences for Gamification Strategies to Motivate Pro-Environmental Behaviour
}

\author{
Leen Van Houdt \\ Department of Computer Science, KU Leuven \\ Leuven, Belgium \\ leen.vanhoudt@kuleuven.be \\ Katrien Verbert \\ Department of Computer Science, KU Leuven \\ Leuven, Belgium \\ katrien.verbert@kuleuven.be
}

\author{
Martijn Millecamp \\ Department of Computer Science, KU Leuven \\ Leuven, Belgium \\ martijn.millecamp@kuleuven.be
}

Vero Vanden Abeele

Department of Computer Science, HCI/eMedia, KU Leuven

Leuven, Belgium

vero.vandenabeele@kuleuven.be

\begin{abstract}
Much topical gamification research has focused on the application of personality trait models and the development of player typologies. These models are often applied under the assumption that personality or gamification user type reside as a stable construct within the individual. However, the preference for gamification strategies as well as their motivational impact, may vary in different contexts and situations. Therefore, our study aimed to disambiguate the different levels of motivation (global, contextual and situational) underlying preferences for gamification strategies, in the specific domain of pro-environmental behaviour. To this end, we developed a gamified mobile app to promote pro-environmental behaviour. Next, 56 participants interacted with the app during on average 22 days, while logs with various game elements were collected. Additionally, participants filled out the Hexad questionnaire to establish gamification user type, a survey polling for intrinsic versus extrinsic motivation towards the environment, and two questionnaires to assess pro-environmental behaviour before and after app usage. Our findings suggest that particularly intrinsic motivation towards the environment predicts preferences for gamification strategies whereas gamification user types fall short. In sum, our study lends support to measuring at different levels of motivation to understand and tailor gamification strategies for pro-environmental behaviour.
\end{abstract}

\section{CCS CONCEPTS}

- Human-centered computing $\rightarrow$ Empirical studies in $\mathrm{HCI}$; User models.

\section{KEYWORDS}

Gamification; Tailoring; User motivation; Pro-environmental behaviour; Hexad

Permission to make digital or hard copies of all or part of this work for personal or classroom use is granted without fee provided that copies are not made or distributed for profit or commercial advantage and that copies bear this notice and the full citation on the first page. Copyrights for components of this work owned by others than ACM must be honored. Abstracting with credit is permitted. To copy otherwise, or republish, to post on servers or to redistribute to lists, requires prior specific permission and/or a fee. Request permissions from permissions@acm.org.

CHI PLAY '20, November 2-4, 2020, Virtual Event, Canada

(C) 2020 Association for Computing Machinery.

ACM ISBN 978-1-4503-8074-4/20/11 . \$ \$15.00

https://doi.org/10.1145/3410404.3414244

\section{ACM Reference Format:}

Leen Van Houdt, Martijn Millecamp, Katrien Verbert, and Vero Vanden Abeele. 2020. Disambiguating Preferences for Gamification Strategies to Motivate Pro-Environmental Behaviour. In Proceedings of the Annual Symposium on Computer-Human Interaction in Play (CHI PLAY'20), November 2-4, 2020, Virtual Event, Canada. ACM, New York, NY, USA, 13 pages. https://doi.org/10.1145/3410404.3414244

\section{INTRODUCTION}

Over the past years, gamification, i.e., the use of game elements in non-gaming contexts [11], has boomed and become an established track in HCI research [48]. Whereas the first wave of gamification research primarily addressed whether it works [39], most recent works attempts to disentangle why. One particular strand in the maturing of gamification research has focused on the application of personality trait models $[20,23,28,54]$ and the development of player typologies $[7,37,38,40,52,54,61]$ to better understand and predict user preferences for specific gamification strategies. This knowledge can inform gamification designers [41] or algorithms [53] to tailor gamified services to idiosyncratic personalities or player styles. The aspiration is that when gamification strategies align with the individual user, this will improve their motivation and promote the overall effectiveness of the gamified app or service. Thus far, studies that have investigated the associations between personality trait and/or player type models and game preferences have not been overly successful, showing modest correlations between user type and game elements, as well as somewhat contradicting results across different domains [20, 23, 54]. It has been argued that simply establishing " $a$ dominant user type is not sufficient to differentiate users according to their preferences for game elements" [20]. Recent editorials of gamification research collections have articulated a need to strengthen gamification studies [39, 44], and in particular to "validate theories of how design elements function and interact with individual dispositions, situational circumstances, and the characteristics of particular target activities" [39].

Indeed, current research based on models to tailor gamification $[31,35,38,54]$ often assumes personality and player type to reside as stable constructs within the individual, irrespective of different contexts (e.g., health, education, marketing) and specific usage situations (e.g., fleeting daily interactions with a smartphone versus dedicated weekly sessions behind a PC). Yet, recent studies suggest that the motivational impact of game elements may vary with the user activity or the domain of the gamified systems $[20,54,59]$. 
A second strand in the need for the maturing of gamification research is "connecting theoretical with applied work" [48]. Many of the aforementioned studies on the tailoring of gamification rely on hypothetical scenarios of using gamification strategies. For example, participants, recruited via a crowdsourcing platform, are asked to imagine a certain usage scenario, are presented illustrations of gamification strategies and next are asked to self-report on their preferences for these strategies [e.g., 20, 40, 41, 59]. While these studies are highly valuable, they are somewhat artificial in setup and exposure to the gamification strategy. Therefore, the findings may be limited in the extent to which they can be transferred to realworld situations. Moreover, the strategies people claim to prefer, may not necessarily correspond with the elements they actually interact with in a gamified application [13]. This may be particularly true in contexts with strong sociocultural norms and influences.

In this paper, we set out to address the aforementioned challenges. Firstly, we aimed to disambiguate different levels of motivational factors underlying preferences for gamification strategies, within the context of pro-environmental behaviour. In recent years, sustainability has become particularly topical, and several studies suggests that gamification can positively influence pro-environmental behaviour [4, 24, 36, 46], both short and long term. However, several studies also highlight the importance of initial attitudes to motivate pro-environmental behaviour [6, 42]. In this study, we devised to investigate associations between pro-environmental behaviour and motivational orientations at three different levels: at the global level (i.e., as stable factor within a person), at the contextual level (i.e., the specific application domain) and at situated level (the usage of a specific gamification features).

Secondly, we aspired to rely on "real-world usage data". To this end, we first designed and developed a smartphone application which encompasses different gamification strategy to motivate and support pro-environmental behaviours. During 22 days, 56 users interacted with the gamified app. During their use, logs of interactions with the different gamification strategies were captured. In addition, users were asked to fill out questionnaires to establish their gamification user type, to assess their contextual motivation for acting pro-environmentally, and to measure their actual proenvironmental behaviour itself. Next,we investigated associations between motivational orientations at the global, contextual, and situated level, and how these predict pro-environmental behaviour.

Our findings support the argument that a stable concept of someone's personality or user type may not be sufficient to predict gamification preferences in the context of pro-environmental behaviour. Instead, our study foregrounds the importance of understanding motivation at the contextual level, and in particular intrinsic motivation, when aiming to understand and predict preferences for gamification strategies. Relating this to the person-situation debate, gamified interventions may be designed more effectively when tailored not only to personality but equally to pre-existing contextual motivations.

\section{BACKGROUND}

In this section, we start with giving a brief overview of models to tailor gamification and relate this to the person versus situation debate. Next, we explain the hierarchical model of motivation and how it may apply to pro-environmental behaviour. We conclude with a summary of research questions and hypotheses.

\subsection{Tailoring gamification strategies based on a personality or player type}

With the maturing of gamification research, one-size-fits-all approaches are increasingly critiqued. Instead, models to tailor gamification to individual differences have gained interest. To this end, various player typologies have been put forward (e.g., Bartle Player types [2], Gamer Motivation Profile [61] or BrainHex [3, 7]). However, to date, only few typologies have been empirically validated. One validated and widely used model is Hexad [32, 52]. With conceptual roots in Self-Determination Theory (SDT) [9], Hexad distinguishes six different user types based on their mapping onto intrinsic and extrinsic motivators, and the basic universal needs (relatedness, autonomy, mastery and purpose). Each of the six gamification user types is also associated with a preferred style of interaction with gameful applications. Socialisers, motivated by relatedness, want to interact with others and create social connections. Free Spirits, motivated by autonomy and self-expression, want to create and explore. Achievers, motivated by mastery, are looking to gain knowledge, learn new skills and improve themselves. Philanthropists, motivated by purpose, are altruistic and want to enrich the lives of other people. Players, motivated by extrinsic rewards, will do what is needed to collect rewards from a system. Finally, the last type, Disruptors, motivated by change, is not derived from SDT, but from empirical observations of online systems [32]. Disruptors want to disrupt the system to force positive or negative change. To measure the extent to which an individual user loads on each of the six user types, Tondello et al. developed and validated a standard 24-item scale for Hexad [52, 54].

Several scholars have investigated the associations between the different user types and game elements [e.g., 20, 41, 54]. Tondello et al. [54], when validating the Hexad scale with undergraduate and graduate students, investigated and confirmed several associations as predicted by Marczewski [32]. Orji et al. [41] specifically investigated associations between the user types and gamification strategies in a health context, with participants that were recruited via Amazon Mechanical Turk. Reported associatons differ from those reported by Tondello. Finally, Hallifax et al. [20] used a crowdsourcing platform as well, and found significant correlations when examining the relationship between Hexad user types and preferences for game elements. Yet, the correlations found were relatively small, and did not fully align with those originally proposed by Tondello et al. [54]. In sum, the studies yielded heterogeneous and somewhat modest outcomes, suggesting differences across application domains, participants and the way in which gamification strategies were operationalised. Noteworthy, the study by Hallifax [20] did find Hexad to outperform other models, in particular the five-factor model of personality (FFM) [35] and BrainHex [3], when investigating preferences for gamification strategies. 
These inconclusive results have led researchers to question whether it is sufficient to simply establish a dominant user type [20] to differentiate users, and tailor gamification strategies. In the specific case of the Hexad scale, the original authors have addressed this issue, stating users might behave differently depending on context [14,33], and urging future research to compare the scale between different contexts [14]. Yet, current interpretations and studies based on Hexad suggest gamification user type to be a stable factor, carrying over in different contexts and situations. When focusing on gamification user type only, and by ignoring the importance of specific contexts and usage situations, "gamification researchers seems blissfully unaware of 40 years of person-situation debate in psychology" as noted by Nacke and Deterding [39].

\subsection{Tailoring gamification strategies based on contextual and situational motivation}

Interestingly, SDT, to date the most prevalent theory in gamification research [48], comes with a "hierarchical model of intrinsic and extrinsic motivation" [57], that highlights the complex interactions between person and situation. In particular, it posits that intrinsic, extrinsic and a-motivations co-exist and interact at three levels of generality: the global level (i.e., personality), the contextual level (i.e., a specific life domain), and the situational level (i.e., performing a specific activity at a specific time) [57, p.44]. At the global level, motivational orientation (intrinsic or extrinsic) is considered most stable. It is a general orientation, built over years, and carries over contexts and situations. Hence, it can be understood as part of one's personality, similar to Hexad. At the contextual level, motivational orientations differ for "distinct spheres of human activity". Hence, motivation at the contextual level is strongly tied to attitudes and affect associated with the specific life domain (for example attitudes towards pro-environmental behaviour). It is also considered moderately stable for that specific life domain. Finally, at the situational level, motivational orientation drives why individuals engage in a specific action at a specific time (e.g., interacting with a gamified app). It is considered most unstable, because of "its responsiveness to the environment".

The hierarchical model, as an integrative framework, also postulates that motivation at a given level results from a combination of 1) top-down motivation at the proximal level and 2) factors in the social environment. In other words, to understand why individuals choose to interact with gamification strategies (the situational level), gamification researchers cannot limit themselves to the global (i.e., personal) level, but should particularly address motivational orientation at the contextual level (e.g., motivations towards the environment). SDT also helps to understand that an understanding of motivations at the different levels is needed to explain behavioural outcomes. [9].

Following up on the hierarchical model, several questionnaires have also been developed to measure and model motivation at the different levels of motivation, such as the Global Motivation Scale [17], or at the contextual level (tailored to specific domains) the Sports Motivation Scale [43] or the Motivation Towards the Environment Scale [6].

\subsection{Tailoring gamification strategies based on internal regulation}

What these questionnaires have in common is that they assess motivational orientation along a continuum, as detailed in SDT's Organismic Integration Theory (OIT) $[8,10]$. OIT highlights that motivation, rather than being binary (motivated or a-motivated), is a continuum. People can be categorised into six different stages along this continuum according to the extent external regulators of motivation are internalised (i.e., intrinsic motivation, integrated regulation, identified regulation, introjected regulation, external regulation, and a-motivation). On one end, intrinsically motivated people have fully internalised the regulations and are motivated to execute specific tasks simply because they find them inherently enjoyable and interesting. On the other end, a-motivated people have no motivation at all to perform a specific task. External regulation is the least autonomous form of motivation, controlled by external regulators only such as obtaining rewards or avoiding punishment [9].

Van Velsen et al. assessed motivation of older adults, according to the level of internalisation and the contextual level (motivations towards self-management of health) [59]. Interestingly, they found intrinsic motivation was associated with a higher appreciation of all persuasive elements, also those hypothesised to tailor to externally regulated motivation only. This suggest that tailoring to internalisation stage and contextual level complements tailoring at the person-level.

2.3.1 Contextual motivations towards the environment. One of the application domains where motivational orientations, both intrinsic and extrinsic, have been researched at length, is pro-environmental behaviour [42], informed by the field of environmental psychology [51]. Well-known strategies, associated with intrinsic motivation, focus particularly on educating people. Knowing more about the environment ("system knowledge") or the consequences of one's own behaviour ("impact knowledge") promotes long-term pro-environmental behaviour [6, 42]. Moreover, it is found critical to empower people and provide them with a sense of ownership [22], people should feel capable of making a difference and should be able to set and achieve goals [49]. Strategies associated with extrinsic motivation rely on providing incentives or reinforcements [42], increasing the cost of engaging in harmful behaviour (e.g., a generating waste or the price of gasoline), while at the same time rewarding and increasing the attraction of environmentally responsible behaviour (e.g., cash for tin cans). These are known to lead to short-term effects in pro-environmental behaviour, but are inadequate to instill long-term change [42].

2.3.2 Motivating pro-environmental behaviour through gamification. The aforementioned strategies lend themselves well to translating to a gamified service or app. Not surprisingly, a growing number of gamification studies have targeted pro-environmental behaviour [24] and/or are concerned with the effect of gamification on sustainable behaviour [29]. Research in the context of energy consumption [18, 24, 27, 36, 46, 50], sustainable nutrition [4], and green transportation habits $[12,15,16,21]$ suggests that gamification and persuasive technology can have a positive effect on pro-environmental behaviour. Some of these works explicitly refer 
to the importance of participants' pre-existing attitudes towards the environment. Berger and Schrader [4] for example, have suggested that gamified interventions should be tailored to "the prior motivation, intentions, and level of self-efficacy of individual users", as these factors influence the effectiveness of gamification strategies. This focus on pre-existing contextual motivations when designing interventions aimed at installing pro-environmental behaviour is also emphasised by Steg and Vlek [51], who argue for factoring in moral beliefs and values, as well as affect, for successful interventions in pro-environmental behaviour.

\subsection{Summarising research objectives and hypotheses}

In sum, to inform the tailoring of gamification strategies towards pro-environmental behaviour, there is a need for a better understanding of the associations between motivational orientations at the global, contextual, and situated level, and how these predict proenvironmental behaviour. In this study, we investigate the following research questions and hypotheses.

2.4.1 Predicting associations with gamified strategies. As a first line of investigations, based on SDT's hierarchical model [57], we hypothesise that a higher motivation at the contextual level is associated with a higher preference for gamification strategies (i.e., the situational level). More particularly, based on [6, 42], we hypothesise that higher intrinsic motivation towards the environment (IMTE) is associated with a higher preference for intrinsically oriented gamification strategies (goals, knowledge, progress). Based on $[42,59]$, we also predict associations between extrinsic motivation towards the environment (EMTE) and those gamification strategies related to incentivization and reducing the cost for engaging in the targeted behaviour [42, p.210].

H1a: IMTE is positively associated with intrinsically oriented gamification strategies.

H1b: EMTE is positively associated with extrinsically oriented gamification strategies.

Moreover, based on earlier work by Hallifax et al. [20] and Tondello et al. [54], we hypothesise a number of associations between Hexad user type (i.e., the global level) and gamification preference. All hypothesised correlations are specified in Table 2.

H1c: Specific gamification user types are associated with specific gamification strategies.

Finally, in line with SDT, which postulates that motivation at a given level results from a combination of 1) top-down motivation at the proximal level and 2) factors in the social environment, we predict associations between gamification preferences and the contextual level (IMTE/EMTE) to be stronger than associations between gamification preferences and the global level (HEXAD).

H1d: Associations between gamification preferences and motivation at the contextual level (IMTE/EMTE) are stronger than associations between gamification preferences and motivation at the global level (HEXAD).

2.4.2 Predicting pro-environmental behaviour.

As a second line of investigations, we also wish to understand the relationships between the different levels of motivation and the desired behavioural outcome: pro-environmental behaviour. At the global level. While we do not have empirical studies to suggest specific hypotheses, on theoretical grounds, i.e., given the roots of Hexad in SDT and the existence of intrinsic versus extrinsic gamification user types, we are interested in exploring associations between gamification user types and pro-environmental behaviour, before and after the gamified app is used.

RQ2: Which associations can be found between gamification user types and pro-environmental behaviour before and after the gamified app is used?

At the contextual level. Based on $[4,42,51]$ we hypothesise that motivation towards the environment, as a moderately stable construct, is related to actual pro-environmental behaviour, before and after the gamified app is used. More specifically, based on [42], it is hypothesised that particularly IMTE positively predicts pro-environmental behaviour, whereas EMTE does not.

H2a: IMTE predicts pro-environmental behaviour before and after the gamified app is used.

H2b: EMTE does not predict pro-environmental behaviour before or after the gamified app is used.

At the situated level. Finally, we expect the preferences for gamification strategies [57, p.44] that are associated with intrinsic motivation $[42,49]$, such as providing knowledge and supporting goals, to predict pro-environmental behaviour, after the use of the gamified app. This is not the case for those gamification strategies that map onto extrinsic motivation, i.e., incentives and rewards.

H2c: Interaction with intrinsically oriented gamification strategies predicts pro-environmental behaviour, after the gamified app is used.

H2d: Interaction with extrinsically oriented gamification strategies does not predict pro-environmental behaviour, after the gamified app is used.

\section{METHODS}

To address our research questions and test our hypotheses, we conducted an in-the-wild study in which participants were asked to install and use a gamified application promoting pro-environmental behaviour for several weeks (22.06 days on average, SD 11.47, ranging from 1 to 47 days). During this usage period, the interaction of users with the application was logged and in addition to these logs, participants completed several questionnaires. The entire procedure was approved by the university's Ethics Committee (dossier no. G- 201902 1522).

\subsection{Gamified application}

The application, developed for this study, can be seen in Figure 1. The design contains four distinct gamification strategies and associated game elements (as described in Table 1): goals via providing actions and daily challenges; knowledge by sharing information, through giving facts on the impact of actions, progress via the implementation of levels, and finally incentivisation by providing points, and by allowing to buy and set new avatars. These strategies and elements are divided over three pages: a homepage with an overview of pro-environmental actions, a profile page with information about the user and avatar, and a page which contains a daily challenge. 


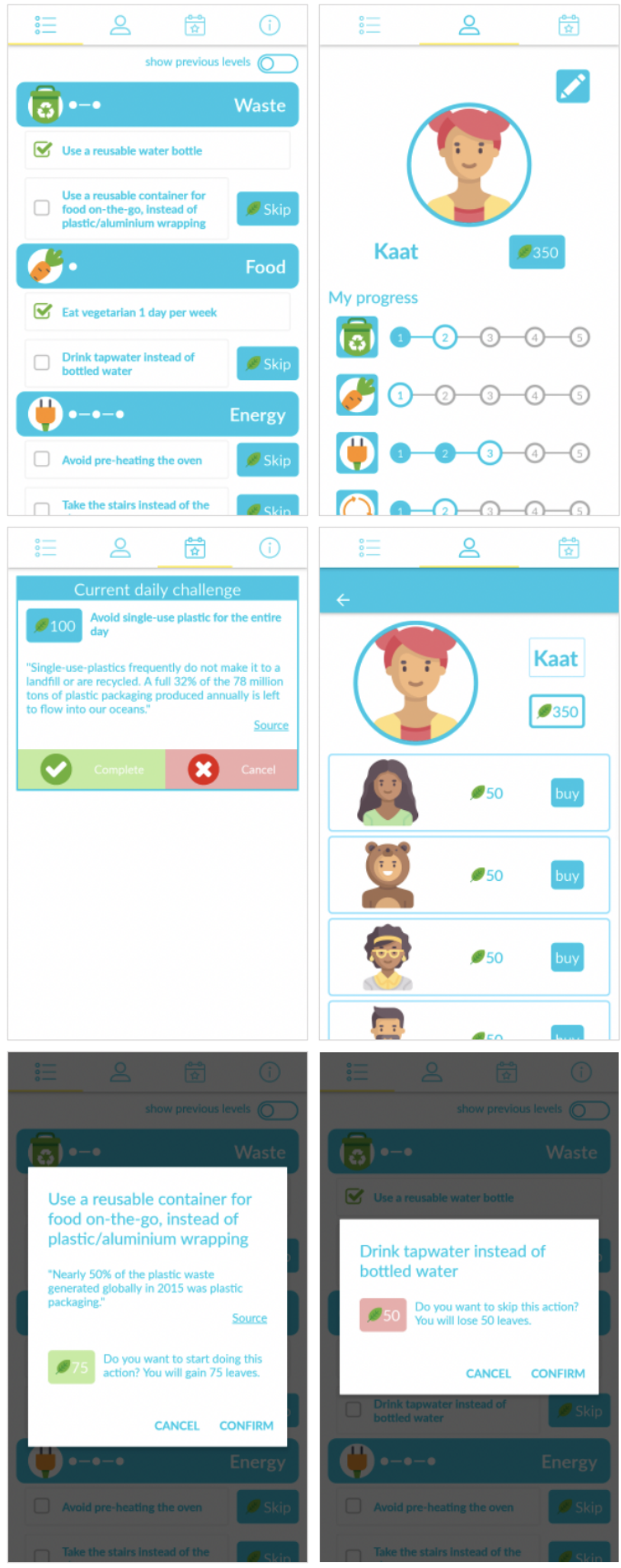

Figure 1: Final application with game elements (left to right, top to bottom): (1 \& 2) levels/progression, (3) daily challenge, (4) customisation, (5) points, and (6) skipping actions.
The homepage shows an overview of pro-environmental actions (e.g., "drink tap water instead of bottled water", "bring a reusable bag to the store"), divided into five categories of behaviour (reducing waste, eating more sustainable food, lowering energy use, decreasing consumption and using green mobility), based on [55, 56, 60]. Each category contains five levels and each level contains two actions. Users can progress within a certain category by completing, hence ticking off actions which will make them gain points, or skipping them, which will make them lose points. When ticking off actions, the application will show a confirmation screen with a fact that emphasises the impact and importance of the action. On the profile page, users can view information about themselves, such as their name and avatar, as well as how many points they currently have, and in which level they find themselves for each category. Through the profile page, users can also customise their avatar, but this will cost them points. Each day, the user is given a new daily challenge which they can complete to earn points. In total there are five challenges, which correspond to the five different categories of behaviour. These challenges are somewhat more difficult than the actions on the homepage, containing prompts such as "avoid single-use plastic for the entire day". Similar to the actions on the homepage, a fact is shown to the user to indicate the impact and importance of the challenge.

Table 1 presents the mapping of logs, game elements and game strategies. Table 2 presents the hypothesised associations between gamification strategies present in the application, and their mapping onto intrinsic or extrinsic motivational orientation, as well as Hexad user type. The rationales for these mappings are the following. The completion of actions, challenges, and levels is considered intrinsically oriented, since these user interactions reflect investment in the knowledge and the setting of pro-environmental goals $[42,49]$. Buying new avatars, the obtainment of points and skipping actions are mapped on an extrinsically oriented gamification strategy.

As regards the six Hexad player types, several correlations with the gamification interactions are hypothesised based on correlation coefficients found in previous work by Hallifax et al. [20] and Tondello et al. [54], who both investigated correlations between the Hexad and gamification preferences at the global level. These previously found correlations coefficients, along with our hypotheses, can also be seen in Table 2.

\subsection{Measures}

3.2.1 Preferences for gamification strategies - Measurement at the situational level. To assess preferences for the different gamification strategies, participants' interactions with the game elements present in the application were logged for the duration of the time they used the application. More specifically, we logged how many times a particular user completed a new sustainable action, skipped an action, completed a daily challenge, levelled up, bought a new avatar, and how many points they gathered. For an overview of the specific numbers, see Table 1.

3.2.2 Gamification user types - Measurement at the global level. The standard 24-item scale for Hexad, developed and validated by 
Table 1: Interaction logs of the 56 participants and the mapping on game elements, gamification strategies and intrinsic versus extrinsic motivation on the basis of $[6,42,49]$

\begin{tabular}{|c|c|c|c|c|c|}
\hline Mot. & Gamification strategies & Game elements & Interaction logs & Mean (SD) & Total \\
\hline $\mathrm{IM}$ & Goals/knowledge & $\begin{array}{l}\text { Adding and completing ac- } \\
\text { tions/Facts on impact ac- } \\
\text { tions }\end{array}$ & Adding an action & $21.18(11.91)$ & 1186 \\
\hline IM & Goals/knowledge & $\begin{array}{l}\text { Daily challenges/Facts on } \\
\text { impact actions }\end{array}$ & Completing a daily challenge & $2.43(3.71)$ & 136 \\
\hline $\mathrm{IM}$ & Progress & Levels & Levelling up & $10.93(7.88)$ & 612 \\
\hline \multirow[t]{2}{*}{ EM } & Incentivisation & Collecting points & Total points & $1381.70(1038.74)$ & 77375 \\
\hline & Incentivisation & Buying a new avatar & New avatar & $0.75(0.74)$ & 42 \\
\hline EM & Reducing barriers & Skip action & Skipping an action & $4.20(7.75)$ & 235 \\
\hline
\end{tabular}

Table 2: Hypothesised correlations between Hexad user type, and motivation towards the environment (intrincic (IM) or extrinsic motivation (EM)) and gamification element preference, based on [42, 49, 59] and correlation coefficients found in previous research $[20,54]$

\begin{tabular}{|c|c|c|c|c|c|c|}
\hline & Add action & Complete challenge & Level up & Points & Buy avatar & Skip action \\
\hline IM & + & + & + & & & \\
\hline \multirow{3}{*}{ EM } & {$[42,49,59]$} & {$[42,49,59]$} & {$[42,49,59]$} & & & \\
\hline & & & & + & + & + \\
\hline & & & & {$[42,49,59]$} & {$[42,49,59]$} & {$[42,49,59]$} \\
\hline \multirow[t]{2}{*}{ Achiever } & & + & + & - & & \\
\hline & & $.463^{* *}[54]$ & $.239^{* *}[54]$ & $-.16^{*}[20]$ & & \\
\hline Philanthropist & & $.212^{* *}[54]$ & & & & \\
\hline Free Spirit & & $.412^{+*}[54]$ & $.204^{* *}[54]$ & & $.198^{* *}[54]$ & \\
\hline Socializer & - & & + & & & \\
\hline & $-.30^{* * *}[20]$ & & $-.16^{*}[20] ; .170^{*}[54]$ & & & \\
\hline Player & & $\stackrel{+}{+}$ & $\stackrel{+}{+}$ & $\stackrel{+}{+}$ & $\stackrel{+}{+}$ & \\
\hline Disruptor & & $.207^{* *}[54]$ & $\begin{array}{c}+ \\
.17^{*}[20]\end{array}$ & & $\frac{+}{.136^{* *}}[54]$ & \\
\hline
\end{tabular}

Tondello et al. [54], was used to determine participants' scores on each of the six Hexad gamification user types.

3.2.3 Motivation towards the environment - Measurement at the contextual level. To assess participants' intrinsic/extrinsic motivation towards the environment, we developed a questionnaire based on the revised Sports Motivation Scale (SMS-II) [43], adopting the method by [59] to assess health motivation. Similar to their approach, we adapted and translated the SMS-II to measure motivation towards the environment. This questionnaire aims to assess motivational orientation along a continuum of six stages, according to the extent external regulators of motivation are internalised, as detailed in SDT's OIT $[8,10]$. Each stage of motivation was assessed using four statements, which had to be evaluated by participants on a 7-point Likert scale (ranging from "strongly disagree" to "strongly agree").

In line with [59], to model the participants based on motivational stage, we first performed an exploratory factor analysis (EFA) on the adapted SMS-II questionnaire. Performing the EFA was done using Principal Axis Factoring (PAF) with Oblimin rotation. With a Kaiser-Meyer-Olkin (KMO) index of .81 and a significant Bartlett's test of sphericity $\chi^{2}=1018,23 ; p<.001$, sampling adequacy was considered good. Initially, eigenvalues suggested the presence of 5 factors, but further inspection showed these factors unfit, and not inline with OIT. Factor analysis for 4 and 3 factors also resulted in 
Table 3: Results of the Exploratory Factor Analysis, 2 factor loadings ( $N=56)$

\begin{tabular}{|c|c|c|c|}
\hline \multirow[t]{2}{*}{ Item } & \multicolumn{2}{|c|}{ Factor } & \multirow[t]{2}{*}{ Communality } \\
\hline & 1 & 2 & \\
\hline \multicolumn{4}{|l|}{ Intrinsic motivation } \\
\hline It gives me pleasure when I can act sustainably. & 0.56 & - & 0.67 \\
\hline I always find it interesting to learn how I can improve my way of living to be more sustainable. & 0.72 & - & 0.43 \\
\hline I enjoy discovering new ways of acting sustainably. & 0.64 & - & 0.75 \\
\hline I find it deeply satisfying to act sustainably. & 0.73 & - & 0.36 \\
\hline \multicolumn{4}{|l|}{ Integrated regulation } \\
\hline Acting sustainably reflects the essence of who I am. & 0.80 & - & 0.43 \\
\hline Through acting sustainably, I am living according to my own principles. & 0.63 & - & 0.90 \\
\hline I find acting sustainably to be one of my core values. & 0.82 & - & 0.77 \\
\hline \multicolumn{4}{|l|}{ Identified regulation } \\
\hline Acting sustainably has become important to me. & 0.87 & - & 0.52 \\
\hline I have come to realize that acting sustainably is necessary. & 0.60 & - & 0.52 \\
\hline I have come to recognize that acting sustainably is important. & 0.68 & - & 0.64 \\
\hline \multicolumn{4}{|l|}{ Introjected regulation } \\
\hline I would feel bad about myself if I did not take the time to act sustainably. & 0.65 & - & 0.37 \\
\hline \multicolumn{4}{|l|}{ External regulation } \\
\hline I mainly act sustainably, because people I care about would be upset if I did not. & - & 0.87 & 0.68 \\
\hline The main reason why I act sustainably is because people around me find it important that I do. & - & 0.94 & 0.58 \\
\hline I act sustainably because I fear others would disapprove of me if I did not. & - & 0.75 & 0.46 \\
\hline My circle of friends sort of enforces me to act sustainably. & - & 0.63 & 0.47 \\
\hline \multicolumn{4}{|l|}{ A-motivation } \\
\hline Acting sustainably is not that important to me. & -0.8 & - & 0.58 \\
\hline I do not really care about acting sustainably. & -0.76 & - & 0.54 \\
\hline
\end{tabular}

$P A F$ with Direct Oblimin rotation, fixed to 2 factors, KMO .81, Bartlett's test of sphericity $\chi^{2}=1018,23 ; p<.001$

either uninterpretable results or unacceptably low communilities and factor loadings. Finally, similar to [59], factor analysis with 2 factors resulted in satisfactory results, with a total explained variance of $56.40 \%$. Items that did not have a factor loading higher than 0.5 for any factor and items with communalities below 0.3 were pruned from the list.

Table 3 shows the factor loadings and communalities for the different items. The first factor, which we will refer to as intrinsic motivation, is a compound of items that initially assessed intrinsic motivation, integrated regulation, identified regulation and introjected regulation. Extrinsic motivation, the second factor, maps very clearly to the questionnaire, consisting of the 4 SMS-II items that assessed external regulation. No separate factor could be found for a-motivation, as these items loaded are highly on the first factor. Cronbach alpha equals 0.91 for the intrinsic motivation factor and 0.85 for the extrinsic motivation factor. Each item shows a item-tototal correlation of at least 0.6 .

In general, participants scored high on intrinsic motivation, with a mean of 5.40 (SD .80), and low on extrinsic motivation with a mean of 2.76 (SD 1.17).

3.2.4 Pro-environmental behaviour. Finally, in order to assess proenvironmental behaviour of participants before and after using the application, we composed a questionnaire following the same structure as the validated General Ecological behaviour (GEB) Scale $[25,26]$ and the Pro-Environmental Behaviour Scale (PEBS) [34]. Similar to these two scales, our questionnaire presented participants with a list of sustainable actions of which they had to indicate how often they executed them on a 3-point scale ("never", "sometimes" or "always"). This list consisted of the same 50 sustainable actions that were recommended to the user in the mobile application.

\subsection{Data analysis}

To investigate the research questions and hypotheses, the relationship between motivational stage and Hexad profiles on one hand, and pro-environmental behaviour and interaction logs on the other hand, were investigated relying on General Linear Model (i.e., bivariate Pearson correlations and simple linear regression). All analyses were carried out with IBM SPSS v26. First, the distribution of this data was examined for normality and outliers. Upon inspection, several of our usage logs of gamification strategies did not follow a normal distribution. Therefore, the decision was made to use a bootstrapping method to assess confidence intervals and estimate significance. Bootstrapping was done on 1000 samples and with Bias Corrected accelerated (BCa). Although we are performing multiple tests, we decided not to correct for a family wise error rate (FWER). While such a correction lowers the chance of a Type I error, given that the aforementioned studies [20,54] report weak correlations, and given our comparatively smaller sample size, this would also increase the chance of a Type II error. Instead, we decided to report effect size $\left(R^{2}\right)$ and confidence intervals, additional to reporting Pearson correlation coefficients, to improve the interpretability of findings, as suggested by Bishara et al. [5]. 
Table 4: Results of bivariate correlation analysis between intrinsic motivation (IM), extrinsic motivation (EM), and Hexad user types and gamification interaction logs. Values in gray are not significant (p>.05).

\begin{tabular}{lcccccc}
\hline & Add action & Complete challenge & Level up & Points & Buy avatar & Skip action \\
\hline IM & $.472^{* *}(\mathrm{H} 1 \mathrm{a} \checkmark)$ & $.224^{*}(\mathrm{H} 1 \mathrm{a} \checkmark)$ & $.290^{*}(\mathrm{H} 1 \mathrm{a} \checkmark)$ & $.543^{* *}$ & -.047 & .003 \\
& $90 \% C I[.227, .599]$ & $90 \% C I[.033, .356]$ & $90 \% C I[.054, .519]$ & $90 \% C I[.406, .671]$ & & $.153(\mathrm{H} 2 \mathrm{~b})$ \\
EM & -.004 & -.016 & .064 & $-.069(\mathrm{H} 2 \mathrm{~b})$ & $.112(\mathrm{H} 2 \mathrm{~b})$ & -.036 \\
\hline Achiever & -.086 & $.168(\mathrm{H} 1 \mathrm{c})$ & $-.053(\mathrm{H} 1 \mathrm{c})$ & $-.004(\mathrm{H} 1 \mathrm{c})$ & .003 & -.140 \\
Philanthropist & .002 & $-.070(\mathrm{H} 1 \mathrm{c})$ & -.081 & .105 & -.032 & -.018 \\
Free Spirit & -.039 & $-.008(\mathrm{H} 1 \mathrm{c})$ & $-.035(\mathrm{H} 1 \mathrm{c})$ & -.063 & $-.144(\mathrm{H} 1 \mathrm{c})$ & -.223 \\
Socializer & $-.129(\mathrm{H} 1 \mathrm{c})$ & -.040 & $-.163(\mathrm{H} 1 \mathrm{c})$ & .016 & -.051 & -.044 \\
Player & -.194 & $-.048(\mathrm{H} 1 \mathrm{c})$ & -.172 & $-.182(\mathrm{H} 1 \mathrm{c})$ & $.024(\mathrm{H} 1 \mathrm{c})$ & $.268^{*}$ \\
Disruptor & $.285^{*}$ & $-.048(\mathrm{H} 1 \mathrm{c})$ & $.310^{*}(\mathrm{H} 1 \mathrm{c} \sqrt{ })$ & .082 & $.133(\mathrm{H} 1 \mathrm{c})$ & $90 \% C I[.031, .472]$ \\
\hline
\end{tabular}

${ }^{*} p<.05$ (1-tailed), ${ }^{* *} p<.01$ (1-tailed)

\subsection{Participants}

Participants were recruited through the personal network of the authors of this work. The first questionnaire, that assessed pro- environmental behaviour before usage, was filled out by 85 participants. The post-usage questionnaire, which assessed pro-environmental behaviour for a second time, was filled out by 72 participants. The motivation scale was completed by 60 participants. When assessing the survey data, there were 57 participants who completed all parts of the survey. Due to a technical error, we needed to exclude one additional participant. This resulted in a final set of 56 participants (32 female, 24 male) with an average age of 26.61 (SD 10.34), ranging from 17 to 58 . These 56 participants interacted with the application for 22.06 days on average (SD 11.47, range 1-47).

\section{RESULTS}

\subsection{Predicting associations for gamification strategies}

4.1.1 H1a \&H1b: Associations between motivation towards the environment and gamification strategies. To investigate assocations, we performed bivariate correlations between IMTE/ EMTE and the interaction logs.

Hypothesis 1a states IMTE to be associated with intrinsically oriented gamification strategies, as specified in Table 2 . The results of the correlation analysis lend partial support to this hypothesis. IMTE is positively associated with "Add action", "Complete challenge" and "Level up". However, IMTE is, surprisingly, also associated with "Points", which we mapped onto the externally oriented gamification strategy of incentivasation.

Hypothesis $1 \mathrm{~b}$ states that EMTE is associated with extrinsically oriented gamification strategies. The results of the correlation analysis do not support this hypothesis. EMTE is not associated with any of the extrinsically mapped interaction logs ("Points", "Buy new avatar" or "Skip action"). The results are displayed in Table 4.

4.1.2 H1c: Associations between Hexad and preferences for gamification strategies. Hypothesis 1c states that Hexad is associated with specific gamification strategies, as specified in Table 2 based on $[20,54]$. Of the 15 hypothesised relationships, only one could be found: between the Hexad user type Disruptor and the "Level up" interaction log. Additionally, two correlations were found that were not hypothesised, also with the Disruptor gamification type: "Add action" and "Skip action". The results are displayed in Table 4.

4.1.3 H1d: Hexad versus Contextual motivation. Finally, hypothesis $1 \mathrm{~d}$ states that associations between gamification preferences and motivation at the contextual level (IMTE/EMTE) are stronger than associations between gamification preferences and the global level (Hexad). For motivation towards the environment, we hypothesised 6 significant correlations and found 4, with an average of .382 . For Hexad, we hypothesised 15 significant correlations and found 3 , with an average correlation of .287 . Hence, both in the ratio of hypothesised versus found number of correlations, and in the average strength of the correlations, our results lend support to this hypothesis.

\subsection{Predicting pro-environmental behaviour}

4.2.1 RQ2 (global level): Associations between gamification user types and pro-environmental behaviour. Additionally, we aimed to explore associations between gamification user types (assessed at the global level) and pro-environmental behaviour, before and after use of the gamified app. While we did not hypothesise any significant associations, Table 6 shows that two user types do. The Philantropist user type is significantly positively correlated with pro-environmental behaviour, both before usage and after usage. The Player type is significantly negatively correlated with proenvironmental behaviour, again both before usage and after usage. While correlations are modest, they remain consistent before and after usage of the gamified application.

4.2.2 H2a \& H2b (contextual level): Motivation towards the environment predicts pro-environmental behaviour. Next, we aimed to verify associations between motivation towards the environment and pro-environmental behaviour, before and after use of the gamified app. In particular, hypothesis 2a states that IMTE predicts proenvironmental behaviour, whereas hypothesis $2 \mathrm{~b}$ states that EMTE does not. The results of the simple linear regression, displayed in Table 6, suggest support for both hypotheses. Intrinsic motivation 
Table 5: Results of bivariate correlation analysis between Hexad user types and pro-environmental behaviour, before and after using the application. Values in gray are not significant $(p>.05)$.

\begin{tabular}{|c|c|c|c|c|c|c|c|c|}
\hline & \multicolumn{4}{|c|}{ Pro-environmental behaviour before usage } & \multicolumn{4}{|c|}{ Pro-environmental behaviour after usage } \\
\hline & \multicolumn{4}{|c|}{ 95\% Confidence interval } & \multirow[b]{2}{*}{$r$} & \multicolumn{3}{|c|}{ 95\% Confidence interval } \\
\hline & $r$ & $p$ & Lower & Upper & & $p$ & Lower & Upper \\
\hline Achiever & .036 & .792 & -.257 & .290 & .035 & .796 & -.217 & .263 \\
\hline Philanthropist & .359 & .007 & .100 & .573 & .307 & .021 & .031 & .516 \\
\hline Free Spirit & .041 & .764 & -.307 & .387 & .065 & .634 & -.270 & .385 \\
\hline Socializer & -.021 & .878 & -.219 & .186 & .036 & .791 & -.154 & .210 \\
\hline Player & -.367 & .005 & -.560 & -.132 & -.375 & .004 & -.582 & -.151 \\
\hline Disruptor & .005 & .971 & -.302 & .328 & -.005 & .972 & -.249 & .268 \\
\hline
\end{tabular}

Table 6: Results of simple linear regression with intrinsic motivation (IM) and extrinsic motivation (EM) as predictors for pro-environmental behaviour, before and after using the application. Values in gray are not significant (p>.05).

\begin{tabular}{|c|c|c|c|c|c|c|c|c|c|c|c|c|c|c|c|c|}
\hline & \multicolumn{8}{|c|}{ Pro-environmental behaviour before usage } & \multicolumn{8}{|c|}{ Pro-environmental behaviour after usage } \\
\hline & \multicolumn{8}{|c|}{ BCa $90 \%$ CI } & \multirow[b]{2}{*}{$r$} & \multirow[b]{2}{*}{$R^{2}$} & \multirow[b]{2}{*}{$\mathrm{F}$} & & & \multirow[b]{2}{*}{ SE } & \multicolumn{2}{|c|}{ BCa $90 \% \mathrm{CI}$} \\
\hline & $r$ & $R^{2}$ & $\mathrm{~F}$ & $p$ & B & SE & Lower & Upper & & & & & & & Lower & Upper \\
\hline IM & .580 & .337 & 27.422 & $<.001$ & 7.361 & 1.219 & 5.073 & 9.819 & .661 & .436 & 41.821 & $<.001$ & 7.817 & 1.375 & 5.418 & 10.530 \\
\hline EM & -.115 & .013 & .730 & .397 & -1.000 & 1.284 & -3.092 & .761 & -.087 & .008 & .410 & .525 & -.710 & 1.236 & -2.577 & 1.243 \\
\hline
\end{tabular}

Table 7: Results of simple linear regression with user interaction with game elements as predictors for pro-environmental behaviour, after using the application. Values in gray are not significant ( $>$ >.05).

\begin{tabular}{lcccccccc}
\hline & \multicolumn{8}{c}{ Pro-environmental behaviour after usage } \\
\hline Predictor & $r$ & $R^{2}$ & $\mathrm{~F}$ & $p$ & $\mathrm{~B}$ & SE & Lower & Upper \\
\hline Add action & .541 & .292 & 22.302 & $<.001$ & .429 & .092 & .261 & .576 \\
Complete challenge & -.020 & $<.001$ & .022 & .882 & -.052 & .354 & -.705 & .547 \\
Level up & .358 & .129 & 7.962 & .007 & .430 & .189 & .162 & .776 \\
Points & .641 & .410 & 37.577 & $<.001$ & .006 & .001 & .004 & .007 \\
Buy new avatar & -.129 & .017 & .918 & .342 & -1.639 & 1.459 & -4.068 & .260 \\
Skip action & -.024 & .001 & .032 & .858 & -.030 & .192 & -.292 & .352 \\
\hline
\end{tabular}

predicts pro-environmental behaviour, both before and after app usage. Extrinsic motivation does not predict pro-environmental behaviour, neither before nor after app usage.

4.2.3 H2c \& H2d (situational level): Preferences for gamification strategies predict pro-environmental behaviour. Hypothesis $2 \mathrm{c}$ states user interaction with intrinsically oriented gamification strategies predicts pro-environmental behaviour, after the gamified app is used, while hypothesis $2 \mathrm{~d}$ states user interaction with extrinsically oriented gamification strategies does not. To test these hypotheses, again simple linear regression analyses were performed, see Table 7. "Add action", "Level up" and "Points" predict pro-environmental behaviour. Contrary to our expectations, "Completing challenge" does not predict pro-environmental behaviours. Hence, hypothesis 1c can not be confirmed.

As predicted by H1d, the extrinsically oriented gamification strategies ("Buy new avatar", "Skip action") do not predict pro-environmental behaviour. Contrary to our expectations, "Points", considered an extrinsically oriented element, is the strongest predictor of proenvironmental behaviour of all. Hence, hypothesis $1 \mathrm{~d}$ can also not be confirmed.

\section{DISCUSSION}

This paper set out to investigate the influence of different levels of motivations on pro-environmental behaviour, by capturing realworld usage data during long-term user interaction with a gamified app. More specifically, we examined associations between motivation at the global level (Hexad user type), the contextual level (motivation towards the environment) and the situated level (user interactions with specific game elements present in our app). 


\subsection{Does motivation at the contextual level matter?}

The study showed that users with a higher level of intrinsic motivation towards the environment (IMTE) interacted more frequently with intrinsically oriented game elements in the app, but not with the extrinsically oriented game elements, with the exception of "Points". Mapped as extrinsically oriented, this game element showed the strongest correlation of all game elements (this particular finding will be scrutinised further below). Contrary to our expectations, no significant association was found between extrinsic motivation towards the environment (EMTE) and any of the three gamification strategies that theoretically mapped on to extrinsic motivation. In all, from our findings we can conclude that intrinsic but not extrinsic motivation at the contextual level matters, when aiming to predict preferences for gamification strategies. Similar results have been found in the field of education, with a recent study by Lavoue et al. [30] showing that tailored gamification only affects learners who are initially more motivated.

The findings of this study also confirm that IMTE positively influences pro-environmental behaviour, both before and after app usage. In contrast, EMTE does not. In fact, we found that intrinsic motivation at this level explained approximately $40 \%$ of the variance in pro-environmental behaviour. For one variable only, and in a context with many confounding variables, this is high number. Yet, it is in line with other prior studies that highlight the importance of prior attitudes for engaging in pro-environmental behaviour [4, 51]. Hence, our study suggest that particularly intrinsic motivation at the contextual level matters when aiming to understand and predict preferences for gamification strategies and behavioural outcomes.

\subsection{Does motivation at the global level matter?}

Only one out of 15 hypothesised correlations was found between the Hexad gamification user types and user interactions with game elements. Additionally, we stumbled on two correlations that did not align with those found in previous work. Hence, this suggest that the use of gamification user types, when considered stable across multiple domains, may not help predict preferences for gamification strategies in the context of pro-environmental behaviour. This finding is in line with Hallifax [20], who concluded that "the dominant user type is not sufficient to differentiate users according to their preferences for game elements". It is important to note that both Tondello and Marczweski, the original authors of Hexad, have stated that user types may differ based on context [14, 33]. Yet, current studies often consider gamification user type to be a stable construct across different domains. Future research should assess whether a more contextual interpretation of the Hexad user types could lead to better predictions regarding gamification preferences. Nevertheless, while no associations were hypothesised, we did find significant correlations between two Hexad user types and pro-environmental behaviour, consistent before and after usage of the app. We found the Philanthropist user type to be positively correlated with pro-environmental behaviour, and the Player user type negatively correlated. Upon further inspection, this may be explained by the fact that the Philanthropist, more than any other user type, is motivated by engaging in actions that have meaning and purpose. As aforementioned, the importance of morals and value for engaging in pro-environmental beliefs is well documented in environmental psychology [51]. Hence, in the life domain of proenvironmental behaviour, meaning and purpose at at global level may carry over with respect to the behaviour itself. The Player user type on the other hand is somewhat of a mirror of the Philanthropist type, as it is a type that only engages in action when external motivators are present, hence the negative association. In this way, despite its exploratory nature, this part of our study offers some insight on the association between Hexad user type and pro-environmental behaviour.

\subsection{Person versus situation}

Overall, our study lends support to the hierarchical model of SDT and in particular the postulate that specifically motivation at the proximal level matters, i.e., motivation at the situational level is mainly influenced by motivational orientation at the contextual level. These finding bring us back to the person versus situation debate. Our findings support the argument that someone's personality or user type, as for example defined by the Hexad, when interpreted as a stable concept that transcends several domains, may not be sufficient to predict gamification preferences. However, paradoxically, when looking not at game interactions but at behavioural outcomes, Hexad's user types of Philanthropist and Player did show up as significant. In conclusion, this lends support to measuring at different levels of motivation: the global, contextual, and situational.

A recent study in the education field by Hallifax et al. [19] supports this conclusion, showing that tailoring gamification to both initial academic motivation (contextual level) and Hexad user type (global level) can significantly increase intrinsic motivation and decrease a-motivation, compared to tailoring based on one level alone. Similarly, a study by Altmeyer et al. [1] in the physical activity domain found that perceived persuasiveness of gameful design elements is influenced by users' motivation towards physical activity, characterised as stage of behavioural change intention. Altmeyer et al. suggest that both Hexad user type and motivation towards physical activity should be considered when personalising gamification elements for persuasive fitness systems.

\subsection{Can gamification make a difference in the context of pro-environmental behaviour?}

However, our findings also suggest that regardless of whether gamification strategies are intrinsically or extrinsically oriented, users with a high level of intrinsic motivation are more likely to appreciate them. Indeed, when calculating correlations with the total number of user interactions, the results show a trend of users with high IMTE using the system more overall $(r=.258, p=$ $.055,95 \% C I[-.060, .525])$, in contradiction to users with high EMTE $(r=.108, p=.426,95 \% C I[-.154, .371])$, although these results do not reach statistical significance.

This is in line with Van Velsen's findings [59], yet challenges gamification efforts to motivate pro-environmental behaviour. It suggests that gamified apps work for those who are already motivated towards pro-environmental behaviour, yet fails to engage and incentivise those users who are mainly extrinsically motivated. The 
challenges of motivating "the unmotivated" towards acting proenvironmentally has already been highlighted by environmental researchers [47]. Moreover, this finding may not be limited to proenvironmental behaviour, and reminds of the well known Matthew effect [45]. The question then remains how we can design gamification interventions as instruments to motivate people who do not yet show this level of intrinsic motivation, as this is where most gains are to be reaped. Future studies to investigate this challenge are therefore recommended.

\section{LIMITATIONS AND FUTURE WORK}

\subsection{The functional significance of points}

In our study, we categorised points as a game strategy that caters to extrinsic motivation. However, we found this game element had the strongest associations with intrinsic motivation of all game elements, as well as with pro-environmental behaviour post app usage. In hindsight, it seems that our categorisation was faulty, and that points were interpreted by users as contingent on their actions, and perhaps as a token of their achievements. Indeed, in the app, total points earned was closely tied to the actions and challenges completed. This finding underscores the importance of better grasping the functional significance (i.e., psychological meaning) of a given game element. This is also highlighted by Van Roy in [58], and put forward in yet another mini-theory that comes with SDT, Cognitive Evaluation Theory [8, p.10]. This theory explains that the ultimate meaning of a game element as controlling (i.e., extrinsic) versus supporting autonomy (i.e., intrinsic) depends on the meaning that the individual user attributes to it.

More generally, the attribution of one specific game element to one motivational strategy for all users may be overly simplistic. Again this finding cautions a one-size-fits-all approach. Moreover, in our study we also equated actual interactions with game elements to preferences. In this manner we hoped to avoid bias and social desirability that is common with self-reporting. At the same time, our approach has its limitations as well. In particular, it is hard to tease out the functional significance through such interactions. Additionally, preferences for game elements cannot simply be reduced to number of interactions. This suggests a need for mixed methods, where the quantitative approach, based on user metrics, is complemented with qualitative data, for example interviews, to give meaning to behaviours.

\subsection{Modelling users based on internalisation}

In our study, we set out to measure users' motivation according to the extent to which they had internalised external regulators of motivation, as put forward by OIT [10]. However, in our study, no six factors could reliably be found that correspond to the six stages of internalisation. Instead, our data showed two factors only: one for what we refer to as intrinsic motivation, which combines intrinsic motivation, integrated, identified and introjected regulation, and one for what we have called extrinsic motivation in this paper. Moreover, no separate factor was found for a-motivation, since two items assessing a-motivation loaded highly (negatively) on the intrinsic motivation factor. This is in line with results found by Van Velsen et al. [59]. Yet, in order to develop a better understanding of contextual motivation according to internalisation stage, there may also be an opportunity for the use of improved measuring instruments. Such a measurement may exist, i.e., the Motivation Towards the Environment Scale (MTES) [6] is designed to measure motivation towards the environment, but was unknown to us at the time of the study. It is our suggestion that further studies concerning motivation in the context of pro-environmental behaviour use this scale.

\subsection{Sample size and statistical significance}

Finally, our in-the-wild study was conducted with a relatively small sample size $(\mathrm{N}=56)$. Therefore, we cannot rule out that the lack of significant correlations may also be attributed to a lack of statistical power. Future research is needed to strengthen our results by conducting the study with a larger number of participants.

\section{CONCLUSION}

This paper investigated the influence of different levels of motivations on pro-environmental behaviour, by capturing real-world usage data during long-term user interaction with a gamified app. This has provided insights on the motivational associations between Hexad user type (global level), motivation towards the environment (contextual level), and user interaction with gamification strategies in the specific context of pro-environmental behaviour (situational level). Our results show that the Hexad model fell short of predicting preferences for gamification strategies. In line with previous findings, dominant Hexad user types only showed limited and modest correlations with gamification user interaction logs. In contrast, intrinsic motivation to act pro-environmentally did predict preferences. Surprisingly, users with a higher level of extrinsic motivation did not show a higher appreciation for extrinsically oriented elements. These results highlight the importance of understanding users' appreciation of gamification strategies within the specific context to which they are applied. Future gamification research on pro-environmental behaviour should therefore measure motivations at different levels, and focus on how to engage particularly these users who are extrinsically motivated more effectively.

\section{ACKNOWLEDGMENTS}

This work is part of the research projects PANACEA Gaming Platform with project number HBC.2016.0177 and Personal Health Empowerment Project with project number HBC.2018.2012 which are financed by Flanders Innovation \& Entrepreneurship.

\section{REFERENCES}

[1] Maximilian Altmeyer, Pascal Lessel, Linda Muller, and Antonio Krüger. 2019. Combining Behavior Change Intentions and User Types to Select Suitable Gamification Elements for Persuasive Fitness Systems. In Persuasive Technology: Development of Persuasive and Behavior Change Support Systems, Harri Oinas-Kukkonen, Khin Than Win, Evangelos Karapanos, Pasi Karppinen, and Eleni Kyza (Eds.). Springer International Publishing, Cham, 337-349.

[2] R. Bartle. 1996. Hearts, Clubs, Diamonds, Spades: Players Who Suit MUDs. Electronically Published. The fournal of Virtual Environments 1, 1 (1996).

[3] Chris Bateman, Rebecca Lowenhaupt, and Lennart Nacke. 2011. Player typology in theory and practice.. In DiGRA Conference.

[4] Verena Berger and Ulf Schrader. 2016. Fostering Sustainable Nutrition behavior through gamification. Sustainability 8, 1 (2016), 1-15. https://doi.org/10.3390/ su8010067

[5] Anthony J. Bishara and James B. Hittner. 2017. Confidence intervals for correlations when data are not normal. Behavior Research Methods 49, 1 (2017), 294-309. https://doi.org/10.3758/s13428-016-0702-8 
[6] Jelle Boeve-de Pauw and Peter Van Petegem. 2017. Because my friends insist or because it makes sense? Adolescents' motivation towards the environment Sustainability (Switzerland) 9, 5 (2017). https://doi.org/10.3390/su9050750

[7] Marc Busch, Elke Mattheiss, Rita Orji, Peter Fröhlich, Michael Lankes, and Manfred Tscheligi. 2016. Player Type Models: Towards Empirical Validation. In Proceedings of the 2016 CHI Conference Extended Abstracts on Human Factors in Computing Systems (San Jose, California, USA) (CHI EA '16). Association for Computing Machinery, New York, NY, USA, 1835-1841. https: //doi.org/10.1145/2851581.2892399

[8] Edward L Deci and Richard M Ryan. 2002. Handbook of Self-Determination Research. (2002).

[9] Edward L. Deci and Richard M. Ryan. 2015. Self-Determination Theory. International Encyclopedia of the Social \& Behavioral Sciences 21 (2015), 486-491. https: //doi.org/10.1016/B978-0-08-097086-8.26036-4 arXiv:arXiv:gr-qc/9809069v1

[10] Ryan R.M. Deci E.L. 1985. Toward an Organismic Integration Theory. In Intrinsic Motivation and Self-Determination in Human Behavior. Springer, Boston, MA, 113-148. https://doi.org/doi.org/10.1007/978-1-4899-2271-7_5

[11] Sebastian Deterding, Dan Dixon, Rilla Khaled, and Lennart Nacke. 2011. From Game Design Elements to Gamefulness: Defining "Gamification". In Proceedings of the 15th International Academic MindTrek Conference: Envisioning Future Media Environments (Tampere, Finland) (MindTrek '11). ACM, New York, NY, USA, 9-15. https://doi.org/10.1145/2181037.2181040

[12] Bothos E., Prost S., Schrammel J., Röderer K., and Mentzas G. 2014 Watch your emissions: Persuasive strategies and choice architecture for sustainable decisions in urban mobility. PsychNology fournal 12, 3 (2014), 107-126. http://www.scopus.com/inward/record.url?eid=2-s2.0$84924736902\{\&\}$ partnerID $=40\{\&\}$ md5=c94ad6e0ebf20398636ff4f2aa5c2764

[13] World Leaders in Research-Based User Experience. [n.d.]. First Rule of Usability? Don't Listen to Users. https://www.nngroup.com/articles/first-rule-of-usabilitydont-listen-to-users/ Library Catalog: www.nngroup.com

[14] Gustavo Fortes Tondello. 2019. Dynamic Personalization of Gameful Interactive Systems. Ph.D. Dissertation. University of Waterloo. http://hdl.handle.net/10012 14807

[15] Jon Froehlich, Tawanna Dillahunt, Predrag Klasnja, Jennifer Mankoff, Sunny Consolvo, Beverly Harrison, and James A. Landay. 2009. UbiGreen: Investigating a Mobile Tool for Tracking and Supporting Green Transportation Habits. In Proceedings of the SIGCHI Conference on Human Factors in Computing Systems (Boston, MA, USA) (CHI '09). Association for Computing Machinery, New York, NY, USA, 1043-1052. https://doi.org/10.1145/1518701.1518861

[16] Sohrab Gharibpour and Veronika Madzinova. 2016. Designing for Sustainable HC : Location-Based Mobile Application for Encouraging Environmental Friendly Ways of Transportation.

[17] Frederic Guay, Marc R Blais, Robert J Vallerand, and Luk G Pelletier. 1999. The global motivation scale. Unpublished manuscript, Université du Québec à Montréal (1999).

[18] Anton Gustafsson, Magnus Bång, and Mattias Svahn. 2009. Power explorer: a casual game style for encouraging long term behavior change among teenagers. ACE '09 Proceedings of the International Conference on Advances in Computer Enterntainment Technology (2009), 182-189. https://doi.org/10.1145/1690388. 1690419

[19] Stuart Hallifax, Elise Lavoué, and Audrey Serna. 2020. To Tailor or Not to Tailor Gamification? An Analysis of the Impact of Tailored Game Elements on Learners Behaviours and Motivation. 216-227. https://doi.org/10.1007/978-3-030-522377_18

[20] Stuart Hallifax, Audrey Serna, Jean-Charles Marty, Guillaume Lavoué, and Elise Lavoué. 2019. Factors to Consider for Tailored Gamification. In Proceedings of the Annual Symposium on Computer-Human Interaction in Play (Barcelona, Spain) (CHI PLAY '19). ACM, New York, NY, USA, 559-572. https://doi.org/10.1145/ 3311350.3347167

[21] E Hedemalm, J Hallberg, AL Kor, Karl Andersson, and Colin Pattinson. 2017. Promoting green transportation via persuasive games. International conference on Sustainable, Ecological Engineering Design for Society (SEEDS) (2017). http: //www.diva-portal.org/smash/record.jsf?pid=diva2:1119550

[22] Harold R. Hungerford and Trudi L. Volk. 1990. Changing Learner Behavior Through Environmental Education. The fournal of Environmental Education 21, 3 (1990), 8-21. https://doi.org/10.1080/00958964.1990.10753743 arXiv:https://doi.org/10.1080/00958964.1990.10753743

[23] Yuan Jia, Bin Xu, Yamini Karanam, and Stephen Voida. 2016. Personality-targeted Gamification: A Survey Study on Personality Traits and Motivational Affordances. In Proceedings of the 2016 CHI Conference on Human Factors in Computing Systems (San Jose, California, USA) (CHI '16). ACM, New York, NY, USA, 2001-2013. https://doi.org/10.1145/2858036.2858515

[24] Daniel Johnson, Ella Horton, Rory Mulcahy, and Marcus Foth. 2017. Gamification and serious games within the domain of domestic energy consumption: A systematic review. Renewable and Sustainable Energy Reviews 73, February 2016 (2017), 249-264. https://doi.org/10.1016/j.rser.2017.01.134

[25] Florian G. Kaiser. 1998. A General Measure of Ecological Behavior. fournal of Applied Social Psychology 28, 5 (1998), 395-422. https://doi.org/10.1111/j.1559- 1816.1998.tb01712.x

[26] Florian G. Kaiser and Mark Wilson. 2000. Assessing People's General Ecological Behavior: A Cross-Cultural Measure. Journal of Applied Social Psychology 30, 5 (2000), 952-978.

[27] Jesper Kjeldskov, Mikael B. Skov, Jeni Paay, and Rahuvaran Pathmanathan. 2012. Using mobile phones to support sustainability: A field study of residential electricity consumption. Proceedings of the 2012 ACM Annual Conference on Human Factors in Computing Systems (CHI '12) (2012), 2347-2356. https://doi.org/10.1145/2208276.2208395

[28] Jonna Koivisto and Juho Hamari. 2014. Demographic differences in perceived benefits from gamification. Computers in Human Behavior 35 (2014), 179-188. https://doi.org/10.1016/j.chb.2014.03.007

[29] Jonna Koivisto and Juho Hamari. 2019. The rise of motivational information systems: A review of gamification research. International fournal of Information Management 45, October 2018 (2019), 191-210. https://doi.org/10.1016/j. ijinfomgt.2018.10.013

[30] Elise Lavoué, Baptiste Monterrat, Michel Desmarais, and Sébastien George. 2019. Adaptive Gamification for Learning Environments. IEEE Transactions on Learning Technologies 12, 1 (2019), 16 - 28. https://doi.org/10.1109/TLT.2018.2823710 (Scimago Q1, ATIEF A+).

[31] Andrzej Marczewski. 2015. Gamification Mechanics and Elements. Even Ninja Monkeys Like to Play: Gamification, Game Thinking \& Motivational Design (2015), $165-177$

[32] Andrzej Marczewski. 2015. User types. Even Ninja Monkeys Like to Play: Gamification, Game Thinking and Motivational Design 1 (2015), 65-80.

[33] Andrzej Marczewski. 2017. The Trouble With Types. https://www.gamified.uk/ 2017/09/13/the-trouble-with-types/. Online, juli 2020.

[34] Gail L. Markle. 2013. Pro-Environmental Behavior: Does It Matter How It's Measured? Development and Validation of the Pro-Environmental Behavior Scale (PEBS). Human Ecology 41, 6 (2013), 905-914. https://doi.org/10.1007/s10745013-9614-8

[35] Robert R. McCrae and Paul T. Costa. 1987. Validation of the Five-Factor Model of Personality Across Instruments and Observers. Fournal of Personality and Social Psychology 52, 1 (1987), 81-90. https://doi.org/10.1037/0022-3514.52.1.81

[36] Luca Morganti, Federica Pallavicini, Elena Cadel, Antonio Candelieri, Francesco Archetti, and Fabrizia Mantovani. 2017. Gaming for Earth: Serious games and gamification to engage consumers in pro-environmental behaviours for energy efficiency. Energy Research and Social Science 29, April (2017), 95-102. https: //doi.org/10.1016/j.erss.2017.05.001

[37] Lennart E. Nacke, Chris Bateman, and Regan L. Mandryk. 2011. BrainHex: Preliminary Results from a Neurobiological Gamer Typology Survey. In Entertainment Computing - ICEC 2011, Junia Coutinho Anacleto, Sidney Fels, Nicholas Graham, Bill Kapralos, Magy Saif El-Nasr, and Kevin Stanley (Eds.). Springer Berlin Heidelberg, Berlin, Heidelberg, 288-293.

[38] Lennart E Nacke, Chris Bateman, and Regan L Mandryk. 2014. BrainHex: A neurobiological gamer typology survey. Entertainment computing 5, 1 (2014), $55-62$.

[39] Lennart E. Nacke and Sebastian Deterding. 2017. The maturing of gamification research. Computers in Human Behavior (2017 2017). https://doi.org/10.1016/j. chb.2016.11.062

[40] Rita Orji, Lennart E. Nacke, and Chrysanne Di Marco. 2017. Towards Personalitydriven Persuasive Health Games and Gamified Systems. In Proceedings of the 2017 CHI Conference on Human Factors in Computing Systems (Denver, Colorado, USA) (CHI '17). ACM, New York, NY, USA, 1015-1027. https://doi.org/10.1145/ 3025453.3025577

[41] Rita Orji, Gustavo F. Tondello, and Lennart E. Nacke. 2018. Personalizing Persuasive Strategies in Gameful Systems to Gamification User Types. In Proceedings of the 2018 CHI Conference on Human Factors in Computing Systems (Montreal QC, Canada) (CHI '18). Association for Computing Machinery, New York, NY, USA, Article 435, 14 pages. https://doi.org/10.1145/3173574.3174009

[42] Luc G. Pelletier. 2002. A motivational analysis of self-determination for proenviromental behaviors. In Handbook of self-determination research. University of Rochester Press, Rochester, NY, US, 205-232.

[43] Luc G. Pelletier, Meredith A. Rocchi, Robert J. Vallerand, Edward L. Deci, and Richard M. Ryan. 2013. Validation of the revised sport motivation scale (SMS-II). Psychology of Sport and Exercise 14, 3 (2013), 329-341. https://doi.org/10.1016/j. psychsport.2012.12.002

[44] Amon Rapp, Frank Hopfgartner, Juho Hamari, Conor Linehan, and Federica Cena. 2019. Strengthening gamification studies: Current trends and future opportunities of gamification research.

[45] D. Rigney. 2010. The Matthew Effect: How Advantage Begets Further Advantage. Columbia University Press, New York.

[46] Michael Ro, Markus Brauer, Kathy Kuntz, Raj Shukla, and Ingo Bensch. 2017. Making Cool Choices for sustainability: Testing the effectiveness of a game-based approach to promoting pro-environmental behaviors. fournal of Environmental Psychology 53 (2017), 20-30. https://doi.org/10.1016/j.jenvp.2017.06.007

[47] Wanda Sass, Jelle Boeve-de Pauw, Vincent Donche, and Peter Van Petegem. 2018. "Why (Should) I Do Something for the Environment?" Profiles of Flemish 
Adolescents' Motivation Toward the Environment. Sustainability 10, 7 (2018), 2579.

[48] Katie Seaborn and Deborah I Fels. 2015. Gamification in theory and action: A survey. International fournal of human-computer studies 74 (2015), 14-31.

[49] K. M. Sheldon and A. J. Elliot. 1999. Goal striving, need satisfaction, and longitudinal well-being: the self-concordance model. Journal of personality and social psychology 76, 3 (03 1999), 482-497. https://search-proquest-com.kuleuven.ezproxy. kuleuven.be/docview/69660489?accountid=17215 Date completed - 1999-06-17; Date created - 1999-04-02; Date revised - 2020-01-10; Last updated - 2020-01-15.

[50] Miyuki Shiraishi, Yasuyuki Washio, Chihiro Takayama, Vili Lehdonvirta, Hiroaki Kimura, and Tatsuo Nakajima. 2009. Using individual, social and economic persuasion techniques to reduce $\mathrm{CO} 2$ emissions in a family setting. Proceedings of the 4th International Conference on Persuasive Technology (2009), 1. https: //doi.org/10.1145/1541948.1541967

[51] Linda Steg and Charles Vlek. 2009. Encouraging pro-environmental behaviour: An integrative review and research agenda. fournal of Environmental Psychology 29, 3 (2009), 309-317. https://doi.org/10.1016/j.jenvp.2008.10.004

[52] Fortes Gustavo Tondello, Alberto Mora, Andrzej Marczewski, and Lennart E Nacke. 2018. Empirical validation of the Gamification User Types Hexad scale in English and Spanish. (2018).

[53] Gustavo F. Tondello, Rita Orji, and Lennart E. Nacke. 2017. Recommender Systems for Personalized Gamification. In Adjunct Publication of the 25th Conference on User Modeling, Adaptation and Personalization (Bratislava, Slovakia) (UMAP '17) ACM, New York, NY, USA, 425-430. https://doi.org/10.1145/3099023.3099114
[54] Gustavo F. Tondello, Rina R. Wehbe, Lisa Diamond, Marc Busch, Andrzej Marczewski, and Lennart E. Nacke. 2016. The Gamification User Types Hexad Scale. In Proceedings of the 2016 Annual Symposium on Computer-Human Interaction in Play (Austin, Texas, USA) (CHI PLAY '16). ACM, New York, NY, USA, 229-243. https://doi.org/10.1145/2967934.2968082

[55] United Nations. [n.d.]. The Lazy Person's Guide to Saving the World.

[56] United Nations Office in Geneva. [n.d.]. 170 actions to transform our world.

[57] Robert J. Vallerand and Catherine F. Ratelle. 2002. Intrinsic and extrinsic motivation: A hierarchical model. In Handbook of self-determination research. University of Rochester Press, Rochester, NY, US, 37-63.

[58] Rob van Roy, Sebastian Deterding, and Bieke Zaman. 2019. Collecting Pokémon or receiving rewards? How people functionalise badges in gamified online learning environments in the wild. International fournal of Human Computer Studies 127, September 2018 (2019), 62-80. https://doi.org/10.1016/j.ijhcs.2018.09.003

[59] Lex van Velsen, Marijke Broekhuis, Stephanie Jansen-Kosterink, and Harm op den Akker. 2019. Tailoring persuasive electronic health strategies for older adults on the basis of personal motivation: Web-based survey study. Fournal of Medical Internet Research 21, 9 (2019), 1-16. https://doi.org/10.2196/11759

[60] World Business Council for Sustainable Development. [n.d.]. The Good Life Goals.

[61] Nick Yee, Nicolas Ducheneaut, Han-Tai Shiao, and Les Nelson. 2012. Through the Azerothian Looking Glass: Mapping in-Game Preferences to Real World Demographics. In Proceedings of the SIGCHI Conference on Human Factors in Computing Systems (Austin, Texas, USA) (CHI '12). Association for Computing Machinery, New York, NY, USA, 2811-2814. https://doi.org/10.1145/2207676. 2208683 